\title{
A Neutron Scattering Study of the Structure of Poly(dimethylsiloxane)-Stabilized Poly(methyl methacrylate) (PDMS-PMMA) Latexes in Dodecane
}

Hallett, James E.; Grillo, Isabelle; Smith, Gregory N.

Published in:

Langmuir

DOI:

10.1021/acs.langmuir.9b03911

Publication date:

2020

Document version

Publisher's PDF, also known as Version of record

Document license:

CC BY

Citation for published version (APA):

Hallett, J. E., Grillo, I., \& Smith, G. N. (2020). A Neutron Scattering Study of the Structure of

Poly(dimethylsiloxane)-Stabilized Poly(methyl methacrylate) (PDMS-PMMA) Latexes in Dodecane. Langmuir, 36, 2071-2081. https://doi.org/10.1021/acs.langmuir.9b03911 


\title{
A Neutron Scattering Study of the Structure of Poly(dimethylsiloxane)-Stabilized Poly(methyl methacrylate) (PDMS-PMMA) Latexes in Dodecane
}

\author{
James E. Hallett,* Isabelle Grillo, and Gregory N. Smith
}

Cite This: Langmuir 2020, 36, 2071-2081

Read Online

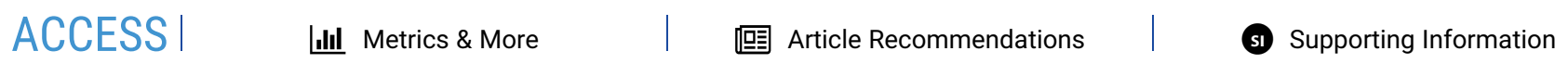

ABSTRACT: Hard-sphere particles in nonpolar solvents are an essential tool for colloid scientists. Sterically stabilized poly(methyl methacrylate) (PMMA) particles have long been used as the exemplary hard-sphere system. However, neither the particles themselves nor the poly(12-hydroxystearic acid) (PHSA) stabilizer necessary to prevent aggregation in nonpolar solvents are commercially available. To counter this, several alternatives have been proposed. In recent years, there has been an increased interest in poly(dimethylsiloxane) (PDMS) stabilizers as a commercially available alternative to PHSA, yet the structure of particles made in this way is not as well understood as those produced using PHSA. In this work, we employ small-angle

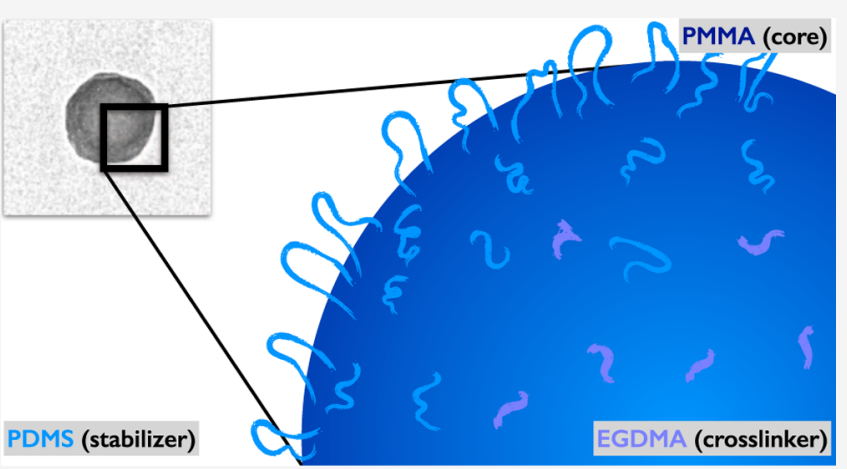
neutron scattering to determine the internal structure of PDMS-

stabilized PMMA particles, synthesized with and without an additional crosslinking agent. We report data consistent with a homogeneous PMMA core with a linearly decaying PDMS shell. The thickness of the shell was in excess of $50 \mathrm{~nm}$, thicker than the PHSA layer typically used to stabilize PMMA but consistent with reports of the layer thickness for similar molecular weight PDMS at planar surfaces. We also show that the amount of the hydrogenous material in the particle core of the crosslinked particles notably exceeds the amount of added ethylene glycol dimethacrylate crosslinker, suggesting some entrapment of the PDMS stabilizer in the PMMA matrix.

\section{INTRODUCTION}

The study of nonpolar poly(methyl methacrylate) (PMMA) colloidal suspensions continues to produce surprising results, more than 30 years since they were first produced as the result of an industrial and academic collaboration between the University of Bristol and ICI. ${ }^{1-3}$ Their utility as a model hard-sphere system has resulted in a vast body of literature studying their dynamic and structural behavior, ${ }^{4}$ while the ability to controllably modify their charge through the addition of surfactants, polymerizable charge groups, or hydrophobic salts has also resulted in many promising applications, particularly in the area of electrophoretic displays.

PMMA particles in nonpolar solvents are usually produced through a one-pot, two-stage dispersion polymerization synthesis, sometimes referred to as the "Antl synthesis". ${ }^{2}$ Typically, methyl methacrylate (MMA), methacrylic acid (MA), the poly (12-hydroxystearic acid) (PHSA) comb stabilizer (as a 30$40 \%$ solution), an initiator, and a chain transfer agent are dissolved in a mixture of hexane and dodecane (or mixture of other alkanes). This mixture is refluxed under nitrogen in a round bottom flask for approximately $2 \mathrm{~h}$ at $80^{\circ} \mathrm{C}$ to grow the polymer particles. The stabilizer is then chemically "locked" to the particle surfaces by heating the mixture to $120{ }^{\circ} \mathrm{C}$ in the presence of diethylamine for approximately $20 \mathrm{~h}$. While there are variations on this, the majority of recent work has relied on modifying this procedure, such as through the formation of a fluorescent core and crosslinked nonfluorescent shell ${ }^{8,9}$ to improve spatial separation in confocal microscopy measurements, or by adding polymerizable charging agents ${ }^{6,10-12}$ to increase their electrophoretic mobility in nonpolar solvents.

Early interparticle scattering work confirmed that PHSAstabilized PMMA nanoparticles in alkanes represented a good model hard-sphere system, even with bimodal size mixtures ${ }^{13,14}$ and at high density. ${ }^{15,16}$ More recently, it has been shown that through the addition of charge additives typically studied for electrophoretic display applications, similar PMMA nanoparticles display different structure at high densities because of their increased repulsion. ${ }^{12}$ Recently, their internal structure was also studied using small-angle neutron scattering (SANS),

Received: December 20, 2019

Revised: January 29, 2020

Published: February 1, 2020 
revealing the localization of absorbed surfactant chargeadditives. ${ }^{17-20}$

The dynamic and structural behavior of PHSA-stabilized PMMA particles have also been widely studied in nonpolar solvents and solvent mixtures, which can be chosen to yield the same refractive index and density as PMMA for fluorescent confocal microscopy applications. However, this can also introduce potentially unwanted particle charge to the system. In bromocyclohexane, the refractive index is nearly matched, and the particles are natively charged. ${ }^{21}$ The magnitude of this charge was shown to be greater when the stabilizer was "locked" to the PMMA matrix. In refractive index and density matching mixtures of cis-decalin and bromocyclohexane, the sign of the charge can be controlled through the choice of fluorescent dye, and can be screened through the addition of the tetrabutylammonium bromide (TBAB) salt. ${ }^{22,23}$ By combining control of the particle charge with the excellent steric stabilization provided by the PHSA stabilizer, complex ionic crystal structures can be produced. ${ }^{24-27}$ Alternatively, for a suspension of particles in a saturated solution of TBAB in cis-decalin and bromocyclohexane, hard-sphere-like behavior can be restored. ${ }^{4,28}$ Nevertheless, this demonstrates that the properties of a supposedly model PMMA suspension can be strongly dependent on small changes to the particle composition.

Despite the wide use of PHSA-stabilized PMMA particles as a model system, whether in alkanes or index-matching solvent mixtures, the PHSA brush copolymer steric stabilizer that is common to all of these particles has proven to be somewhat fickle to produce ${ }^{29}$ and is not commercially available. Although silicone-based stabilizers for PMMA particles in nonaqueous solvents were introduced at a similar time to PHSA, such as in the early work by Croucher and Milkie ${ }^{30}$ and Pelton et al., ${ }^{31,32}$ it is only relatively recently that poly(dimethylsiloxane) (PDMS)based stabilizers have been heralded as a cheaper, more reproducible, and (crucially) commercially available alternative. Klein et al. ${ }^{33}$ proposed PDMS stabilizers with methacryloxypropyl groups at both ends as an alternative to PHSA in the synthesis of PMMA particles and reported highly uniform particles of diameter $0.4-1.5 \mu \mathrm{m}$ with good stability in a range of alkanes and cyclic aliphatics. Richez et al. $^{34}$ extended this approach to other polymers copolymerized with PMMA in the particle cores, and to monomethacryloxypropyl-terminated PDMS and reported similar particle sizes and stabilities. More recently Kodger et al. ${ }^{35}$ developed a PDMS-graft-PMMA comb stabilizer that was used in the synthesis of fluorescent PMMA particles with excellent long-term size stability and negligible dye leakage, ideal for experiments that require long-duration observation. Smaller PMMA particles stabilized by physically adsorbed polystyrene-PDMS block copolymers have also been reported, ${ }^{36,37}$ although these have been found to be less stable in alkanes than those with grafted PMMA-graft-PDMS stabilizers. $^{38}$

Nevertheless, beyond papers describing the method for using PDMS-based stabilizers, there are relatively few reports that use these particles instead of conventional PHSA-stabilized PMMA. Lin et al. ${ }^{39}$ studied the electrophoretic response of PDMSstabilized PMMA in a microfluidic channel, in pure solvent, and in the presence of the AOT surfactant. They reported nonzero electrophoretic mobility even in the absence of the surfactant. With the added surfactant they also observed an electrophoretic mobility which decreased with time, which they interpreted as due to surfactant micelles screening the internal field in their microfluidic cell. Tsurusawa et al. ${ }^{40}$ also used PDMS-stabilized
PMMA particles and induced gelation by adding nonadsorbing polymer to the solvent and suppressing the particle charge with added salt. Thus, while PDMS-stabilized PMMA shows promising behavior, the degree to which these systems have been characterized is limited compared to conventional PHSAstabilized PMMA.

In this work, we report the first use of SANS measurements to study a system of PDMS-stabilized PMMA colloids. This extends the body of work using small-angle scattering to study PMMA latexes ${ }^{12,15-20}$ that was previously limited to the PHSAstabilizer. We do this by synthesizing PMMA latexes using covalently linked PDMS stabilizers to produce smaller colloids than previously reported ${ }^{33,34}$ with a goal of accessing a size regime suitable for small-angle scattering measurements. We compare the particle sizes obtained from scattering with those from electron microscopy and dynamic light scattering (DLS) to show the relationship between the composition and particle size. Because of the earlier reports of some stability issues for smaller PMMA particles, ${ }^{2}$ we also use ethylene glycol dimethacrylate (EGDMA) as a crosslinker, which has been shown to improve PMMA particle stability in various solvents. ${ }^{8,41} \mathrm{We}$ then compare the internal structure of crosslinked ${ }^{42}$ and noncrosslinked particles. Intriguingly, we detect other hydrogenous material in the particle core, which we attribute to internalized PDMS stabilizer.

These results provide useful structural insights into the internal structure of PDMS-PMMA nanoparticles as they become more prevalent in experiments of physical, colloidal interactions. More generally, this report further demonstrates the sensitivity of SANS as a technique to quantitatively describe structural heterogeneity in polymeric latexes and could be used to investigate time or temperature-induced polymer phase separation in, for example, composite particles designed to yield a particular refractive index, where any inhomogeneity would result in inferior optical properties or to determine localization of other additives (such as charge control additives, fluorescent dyes, or crosslinking agents) that are normally assumed to be homogeneously distributed in PMMA particles.

\section{MATERIALS AND METHODS}

Materials. MMA was purchased from Sigma, and deuterated MMA $\left(\mathrm{MMA}-d_{8}\right)$ was purchased from Cambridge Isotope Laboratories. Both MMA and MMA- $d_{8}$ were run through a basic alumina column to remove the inhibitor. Deuterated dodecane (dodecane- $d_{26}$ ) was purchased from Cambridge Isotope Laboratories. Methacryloxypropyl-terminated PDMS (PDMS-MA) $\left(25 \mathrm{~kg} \mathrm{~mol}^{-1}\right), 2,2^{\prime}$-azobisisobutyronitrile (AIBN), octanethiol, MA, EGDMA (all Sigma), hexane (VWR), and dodecane (Alfa-Aesar) were all used as received.

Particle Synthesis. For the noncrosslinked PMMA particles, a procedure was used adapted from previously reported methods, ${ }^{3,34}$ from which we note three general trends: for a given monomer concentration low molecular weight $\left(5 \mathrm{~kg} \mathrm{~mol}^{-1}\right)$ stabilizer results in particles that are unstable, ${ }^{33}$ a higher proportion of stabilizer-tomonomer results in smaller particles, and a higher proportion of monomer to total reaction volume results in larger particles. ${ }^{33,34} \mathrm{We}$ therefore prioritize a higher molecular weight stabilizer $\left(25 \mathrm{~kg} \mathrm{~mol}^{-1}\right)$ at a relatively low monomer concentration (10-25\%), and explore a wide range of stabilizer to monomer ratios to obtain different particle sizes. Typically, a PDMS-MA stabilizer solution in dodecane in a $100 \mathrm{~mL}$ round bottom flask was degassed via nitrogen bubbling for $30 \mathrm{~min}$, and then heated to $80{ }^{\circ} \mathrm{C}$ under magnetic stirring. Simultaneously, a solution of AIBN in MMA (or MMA- $d_{8}$ ) was made, to which dodecane and octanethiol were added. This was also degassed by nitrogen bubbling in an ice bath for $30 \mathrm{~min}$. The monomer solution was then rapidly added to the stabilizer solution via a syringe. The reaction was 
Table 1. Details of the Reagents Used to Produce PDMS-Stabilized PMMA Particles (Amounts in Grams) ${ }^{a}$

\begin{tabular}{|c|c|c|c|c|c|c|}
\hline & $\mathrm{DX}_{242}$ & $\mathrm{D}_{243}$ & $\mathrm{HX}_{253}$ & $\mathrm{H}_{252}$ & $\mathrm{H}_{501}$ & $\mathrm{H}_{1357}$ \\
\hline & PMMA- $d_{8}$ linked & PMMA- $d_{8}$ & PMMA linked & PMMA & PMMA & PMMA \\
\hline \multicolumn{7}{|c|}{ Vessel } \\
\hline MMA- $d_{8}$ & 1.083 & 1.080 & & & & \\
\hline MMA & & & 1.030 & 1.030 & 2.059 & 5.610 \\
\hline dodecane & 7.5 & 7.5 & 7.5 & 7.5 & 15 & 15 \\
\hline AIBN & 0.011 & 0.011 & 0.011 & 0.011 & 0.022 & 0.064 \\
\hline octanethiol & 0.013 & 0.014 & 0.013 & 0.013 & 0.027 & 0.064 \\
\hline PDMS-MA & 0.505 & 0.501 & 0.511 & 0.489 & 0.416 & 0.160 \\
\hline \multicolumn{7}{|c|}{ Syringe } \\
\hline EGDMA & 0.020 & & 0.020 & & & \\
\hline dodecane & 0.15 & & 0.15 & & & \\
\hline
\end{tabular}

${ }^{a}$ Reagents in the reaction vessel and added via syringe as indicated. Particle naming protocol indicates MMA (H) or MMA- $d_{8}$ (D), presence of EGDMA crosslinking agent $(\mathrm{X})$, and number denotes solvodynamic particle diameter in nanometers obtained via DLS (details below).

stirred at approximately $300 \mathrm{rpm}$ and allowed to proceed for $4 \mathrm{~h}$ under nitrogen. For the crosslinked particles, ${ }^{8,9}$ a solution of EGDMA in dodecane was slowly added to the reaction mixture via syringe pump (to avoid coagulation ${ }^{42}$ ) over approximately $45 \mathrm{~min}$, starting as soon as the reaction was started. After the EGDMA solution had been completely added to the reaction mixture, it was also allowed to continue for a total of $4 \mathrm{~h}$.

Upon completion, the reaction mixture was allowed to cool to room temperature and filtered through glass wool to remove any large aggregates. To remove unreacted monomers, the suspension was washed via repeated centrifugation and redispersion in fresh hexane before finally being transferred to dodecane (Table 1).

Dynamic Light Scattering. DLS measurements were performed using a Malvern Zetasizer Nano ZS (Malvern Instruments, UK). Dilute dispersions $(\sim 0.1 \%)$ were studied at $25^{\circ} \mathrm{C}$. Approximately 10 runs of $10 \mathrm{~s}$ duration were performed per measurement. (The exact number was selected by instrument software). Three measurements were performed and averaged.

The wavelength of the radiation was $\lambda=632.8 \mathrm{~nm}$ and the scattering angle was $173^{\circ}$. For a light-scattering measurement, the modulus of the momentum transfer vector $\vec{Q}$ is calculated from $\theta$ (half the scattering angle), $n$ (the refractive index), and $\lambda$. ( $Q$ has the value $0.003 \AA^{-1}$ for these values).

$$
Q=\frac{4 \pi n \sin \theta}{\lambda}
$$

Solvodynamic $Z$-average particle diameters were determined from cumulants analysis (Malvern Zetasizer software). Diffusion coefficients $(D)$ were converted to particle radii $(r)$ using the Stokes-Einstein equation, ${ }^{43,44}$ where $k_{\mathrm{B}} T$ is the thermal energy, and $\eta$ is the solvent viscosity, which is taken from the literature. ${ }^{45}$

$$
D=\frac{k_{\mathrm{B}} T}{6 \pi \eta r}
$$

Additionally, intensity-weighted size distributions were determined using the same software.

Electron Microscopy. Dispersions of small latexes were diluted to generate $0.01 \%$ dispersions. Copper transmission electron microscopy (TEM) grids (Agar Scientific, UK) were surface-coated to yield a thin film of amorphous carbon. Each nanoparticle dispersion was placed onto a grid, and the solvent was allowed to evaporate slowly at room temperature. To stain the deposited nanoparticles, the grids were exposed to ruthenium(IV) oxide vapor for $7 \mathrm{~min}$ at $20^{\circ} \mathrm{C}$ prior to analysis. ${ }^{46}$ This heavy metal compound acted as a positive stain to improve contrast. Imaging was performed at $100 \mathrm{kV}$ using a Phillips CM100 instrument equipped with a Gatan 1k CCD camera.

Samples for scanning electron microscopy (SEM) were prepared by drop-casting dilute dispersions $(0.01 \mathrm{wt} \%)$ in hexane on freshly cleaved mica substrates which were attached to SEM stubs. After allowing the solvent to evaporate they were subsequently sputter-coated with a thin layer of gold. Measurements were performed using a JEOL JSM 6330F SEM.

Number-weighted particle size distributions were obtained using Image 1.51 u. ${ }^{47}$ The area of at least 400 nanoparticles was measured from EM images, before calculating a histogram with $20 \mathrm{~nm}$ wide bins for both TEM and SEM. These histograms were then fit to a log-normal distribution. These were converted to volume-weighted particle size distributions by scaling each bin by the sphere volume and renormalizing. These processed histograms were then also fit to a log-normal distribution.

Neutron Scattering. SANS measurements were performed using the D33 instrument at the Institut Laue-Langevin (ILL, Grenoble, France). Three instrument configurations were used, covering high, middle, and low $Q$. The modulus of the momentum transfer vector $\vec{Q}$ is calculated from $\theta$ (half the scattering angle) and $\lambda$ (the neutron wavelength):

$$
Q=\frac{4 \pi \sin \theta}{\lambda}
$$

For the high- $Q$ and middle- $Q$ configurations, neutrons with $\lambda$ of $6 \AA$ were used, and for the low- $Q$ configuration, neutrons with a $\lambda$ of $13 \AA$ were used. For the low- $Q$ configuration, a sample-to-detector distance of $12 \mathrm{~m}$ and collimation of $12.8 \mathrm{~m}$ were used to give a $Q$ range of $0.0015-0.019 \AA^{-1}$. For the middle- $Q$ configuration, a sample-todetector distance of $13 \mathrm{~m}$ and collimation of $12.8 \mathrm{~m}$ were used to give a $Q$ range of $0.0035-0.039 \AA^{-1}$. For the high- $Q$ configuration, a sampleto-detector distance of $2 \mathrm{~m}$ and collimation of $5.3 \mathrm{~m}$ were used to give a $Q$ range of $0.022-0.47 \AA^{-1}$. The beam size was $7 \times 10 \mathrm{~mm}^{2}$. Data were placed on an absolute scale $\left(\mathrm{cm}^{-1}\right)$ by using a $1 \mathrm{~mm}$ thick sample of liquid water $\left(\mathrm{H}_{2} \mathrm{O}\right)$ as a standard. Raw data were integrated, normalized with respect to transmission and intensity, and stitched together using instrument-specific software, LAMP. During the data reduction, the error in $Q(\mathrm{~d} Q)$ was calculated, and fitted data had $\mathrm{d} Q$ smearing applied.

Data have been fit to models as described in the text using the SasView 4.2.2 small-angle scattering software package. ${ }^{48}$ Data were fit using either a single model or as a linear combination of two models. In the latter case, the cross terms were neglected for computational simplicity and as the scale of the two contributions were sufficiently different to make these terms insignificant. The data at high $Q$ (background and model for PDMS at the interface) was fit first and subtracted from the experimental scattering curve, and the model for the latex fit to the residual scattering at low $Q$.

The quality of the fits were assessed in two ways. Error-weighted residual difference plots $\left(\Delta I / \sigma \equiv\left(I_{\exp }-I_{\text {fit }}\right) / \sigma\right)$, where $I_{\exp }$ and $I_{\text {fit }}$ are the experimental and fit scattering intensities, respectively, and $\sigma$ is the uncertainty of the experimental scattering intensity, were calculated for every value of $Q$ and for every set of fit data and are shown alongside the fits over the entire $Q$ range. ${ }^{49}$ The reduced $\chi^{2}$ (called $\left.\chi_{\mathrm{R}}{ }^{2}\right)$ is also calculated for each set of fit data or combination of sets of data for constrained fits. It is calculated by normalizing by the number of points 
$\left(n_{\mathrm{pt}}\right)$ and the number of fit parameters $\left(n_{\mathrm{par}}\right),\left(\chi_{\mathrm{R}}^{2}=\sum_{i}\left[\left(\left(I_{\exp }\right)_{i}-\right.\right.\right.$ $\left.\left.\left.\left.\left(I_{\text {fit }}\right)_{i}\right)\right)^{2} / \sigma_{i}^{2}\right] /\left[n_{\mathrm{pt}}-n_{\mathrm{par}}\right]\right) .^{48}$

The scattering length densities (SLDs, $\rho_{\mathrm{n}}$ ) of components were calculated using the known mass density $\left(\rho_{\mathrm{m}}\right)$ and stoichiometry. Values for the materials used in the study are shown in Table 2. Mass

Table 2. Mass Density $\left(\rho_{\mathrm{m}}\right)$ and Neutron Scattering Length Density $\left(\rho_{n}\right)$ for All Compounds Used

$\begin{array}{lcc} & \rho_{\mathrm{m}} /\left(\mathrm{g} \mathrm{cm}^{-3}\right) & \rho_{\mathrm{n}} /\left(10^{-6} \AA^{-2}\right) \\ \text { dodecane } & 0.75 & -0.46 \\ \text { dodecane- } d_{26} & 0.864 & 6.71 \\ \text { PDMS } & 0.970 & 0.06 \\ \text { PMMA } & 1.188 & 1.07 \\ \text { PMMA- } d_{8} & 1.255 & 6.86 \\ \text { poly(EGDMA) } & 1.19 & 1.35\end{array}$

densities are taken for the literature, except for the polymer of EGDMA [denoted as poly(EGDMA)], whose density is calculated as the product of the ratio of densities of the PMMA polymer and MMA monomer and EGDMA monomer, assuming that the monomer would be as densified as MMA by polymerization. ${ }^{50-53}$ Neutron scattering lengths were taken from the literature. ${ }^{54}$ The SLDs of the solvent mixtures for contrast variation measurements were modified by mixing dodecane with dodecane- $d_{26}$. The two solvents were assumed to mix ideally, and the scattering length density $\left(\rho_{i}\right)$ of the mixture is equal to the volume fraction $\left(\phi_{i}\right)$ weighted sum of that of each component (calculated using the chemical composition and mass density).

$$
\rho_{\mathrm{t}}=\sum_{i} \phi_{i} \rho_{i}
$$

\section{RESULTS AND DISCUSSION}

In this section, we proceed to characterize the size, shape, and structure of PDMS-stabilized PMMA particles over a range of length scales. By electron microscopy, particle morphology and nonsolvated (dry) particle size was obtained. This was compared to DLS measurements, which yield the solvodynamic size from the particle core and solvated stabilizer layer. We then employ SANS to interrogate the internal structure of the particles and determine the spatial distribution of the stabilizer and crosslinking agent by varying the solvent SLD and using a deuterated PMMA core.

Particle Size. Particles were synthesized over a range of sizes to investigate the role of the stabilizer in increasing the solvodynamic diameter. Large particles of diameter in the range $400-1500 \mathrm{~nm}$ were obtained, comparable to previous reports. $^{33,34}$ By increasing the ratio of stabilizer to monomer, particle core diameters below $200 \mathrm{~nm}$ were also obtained, sufficiently small to perform neutron scattering measurements. We note that the size distributions are broader for the smaller particles than for the larger particles. Similar behavior has been reported previously for PMMA with various steric stabilizers, ${ }^{33,55}$ and was attributed to a wider window for particle nucleation occurring at higher stabilizer concentrations, broadening the size distribution for a given growth rate as earlyforming nuclei will be notably larger than late-forming nuclei. At lower stabilizer concentrations, late-forming nuclei are captured by larger particles before they can become sufficiently sterically stabilized, ${ }^{56}$ but with a higher stabilizer concentration these nuclei are more likely to adsorb enough of the stabilizer to become sterically stable, broadening the size distribution of the dispersion. Electron microscopy (TEM for the small latexes and SEM for the larger latexes) images are shown in Figure S1. These images show that the particles have a spherical morphology and have moderate distribution of sizes. Particle size distributions measured using DLS and electron microscopy are shown in Figure 1 , and the mean diameter values are given in Table 3 . The

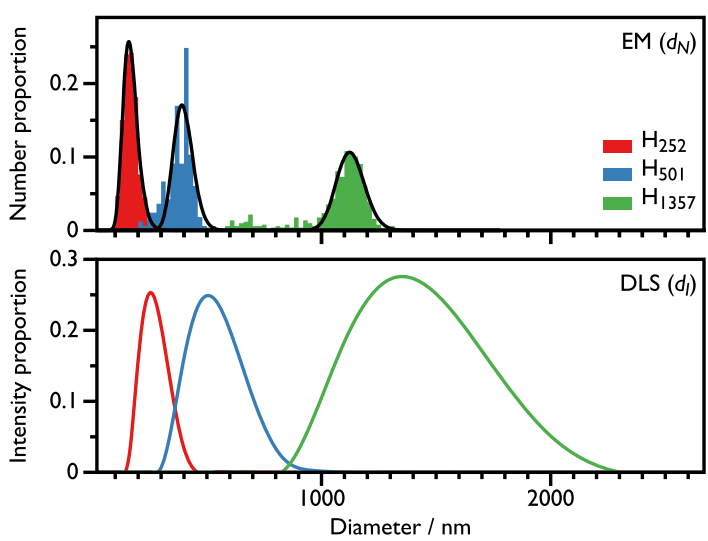

Figure 1. Particle size distributions obtained for PDMS-stabilized PMMA latexes from a variety of methods: DLS and either TEM or SEM, depending on the particle size.

Table 3. Size of Isotopically Labeled Latexes ${ }^{a, b}$

$\begin{array}{lcccc}\text { latex } & \begin{array}{c}\mathrm{DLS} d_{\mathrm{Z}} / \mathrm{nm} \\ (\sigma)\end{array} & \begin{array}{c}\mathrm{EM} d_{\mathrm{N}} / \mathrm{nm} \\ (\sigma)\end{array} & \begin{array}{c}\mathrm{EM} d_{\mathrm{V}} / \mathrm{nm} \\ (\sigma)\end{array} & \begin{array}{c}\text { SANS core } d_{\mathrm{V}} / \mathrm{nm} \\ (\sigma)\end{array} \\ \mathrm{H}_{1357} & 1357(0.24) & 1126(0.05) & 1136(0.05) & \\ \mathrm{H}_{501} & 501(0.20) & 396(0.11) & 408(0.09) & \\ \mathrm{H}_{252} & 252(0.20) & 165(0.19) & 183(0.18) & 179(0.20) \\ \mathrm{D}_{243} & 243(0.11) & 149(0.17) & 163(0.18) & 160(0.20) \\ \mathrm{HX}_{253} & 253(0.16) & 155(0.16) & 170(0.18) & \\ \mathrm{DX}_{242} & 242(0.14) & 139(0.19) & 158(0.21) & 150(0.20)^{c}\end{array}$

${ }^{a} \mathrm{H}$ latexes have PMMA cores, and D latexes have PMMA- $d_{8}$ cores. Only X latexes are crosslinked. Values are considered significant to the nearest $\mathrm{nm} .{ }^{b} d_{\mathrm{Z}}$ is the $Z$-average diameter and $\sigma$ the standard deviation (determined from the polydispersity index), both from DLS measurements. The diameters $(d)$ are the median from log-normal fits to the data, and $\sigma$ is the standard deviation of the natural log of the distribution. These are either number-weighted $\left(d_{\mathrm{N}}\right)$ or volumeweighted $\left(d_{\mathrm{V}}\right)$, as specified. ${ }^{c}$ This is the value for the model with a constant core SLD. If a varying core SLD is used instead, $d_{\mathrm{V}}$ is 173 $\mathrm{nm}$, and $\sigma$ is 0.21 .

intensity weighted diameters measured using DLS are shown along with the number-weighted diameters measured using EM. The DLS size distributions shown in Figure 1 are notably larger than those measured with electron microscopy, by approximately $100 \mathrm{~nm}$. This is not surprising; we expect the solvated PDMS stabilizer layer to be extended and contribute to the solvodynamic diameter for the DLS measurements but to be collapsed and with little contrast in the electron microscopy measurements. Previous studies of the thicknesses of grafted PDMS layers, either solvated or in air, report values of up to 50 $\mathrm{nm}$ for similar molecular weight PDMS stabilizers, ${ }^{57,58}$ so our observations are broadly consistent with this. Furthermore, DLS is biased toward larger diameters than EM because of the relative size weighting of intensity and number averaging, ${ }^{59,60}$ as larger particles scatter more strongly than smaller particles and therefore bias the intensity distribution to larger sizes. Recent measurements also showed a similar discrepancy between electron microscopy measurements and DLS for poly(stearyl methacrylate) - poly(benzyl methacrylate) nanoparticles, ${ }^{61}$ where the (lower molar mass and smaller radius of gyration) polymeric stabilizer also similarly enhance the solvodynamic 
diameter. One might suggest that DLS is more sensitive to particle aggregation (which is difficult to identify in dried electron microscopy samples), and this is the cause of the discrepancy between the two distributions. However, the compatibility between the measured electron microscopy and SANS diameters (discussed below, see Table 3) and the excellent fit to a dilute (with no interparticle structure) SANS model indicate that this is not the case for the smaller latexes, although some aggregation could contribute to the distributions for $\mathrm{H}_{501}$ and $\mathrm{H}_{1357}$.

The size distribution of the smallest noncrosslinked hydrogenated particle system $\mathrm{H}_{252}$ is somewhat broader than equivalent PHSA-stabilized nanoparticle suspensions (where $\sigma$ $\approx 0.1$ for $\sim 100 \mathrm{~nm}$ diameter latexes is typical). ${ }^{19}$ Despite this, features in the neutron-scattering data from this system are sufficient to fit a form factor and to obtain a particle size distribution.

SANS measurements were performed on a dilute dispersion of PDMS-stabilized latexes $\left(\mathrm{H}_{252}\right)$ in unlabeled, hydrogenous dodecane. The scattering data can be well described using a spherical form factor $(P(Q)$ ), given in eq 5 below (SasView sphere model ${ }^{62}$, where $r$ is the radius of the sphere, which is fit with a log-normal size distribution. ${ }^{63,64}$

$$
I(Q) \propto P(Q)=\left[\frac{\sin (Q r)-Q r \cos (Q r)}{(Q r)^{3}}\right]^{2}
$$

For SANS data, the scattering from the PMMA core dominates, and there is no significant contribution from the PDMS stabilizer layer. This is essentially the same as for PHSAstabilized latexes, where the PMMA core dominates unless the solvent scattering length density is matched to the PMMA core. ${ }^{19}$ This simpler form factor is preferred to avoid overparameterizing the data fitting. An attempt to fit the data to a sphere model with a shell did not provide any additional information. The model fit the data best when the SLD of the shell matched that of the solvent, which would be expected for a particle with a highly solvated steric stabilizer layer. ${ }^{19}$

From the fit to the data, the radius of the PMMA core of the latex and the width of the size distribution can be determined. The particle diameter was found to be $178 \mathrm{~nm}$, giving good agreement with the value obtained by TEM but smaller than that obtained via DLS. This is consistent with previous reports that have utilized small-angle scattering methods and DLS to study latexes. ${ }^{19,65} \mathrm{~A}$ comparison between the size distributions obtained from DLS and SANS is shown in Figure 2.

Particle Structure. We now consider the effect of adding a crosslinking agent on the internal particle structure. Crosslinkers serve a key purpose in the synthesis of PMMA; they are known to aid the stability of dispersions in various solvents that would otherwise dissolve or aggregate the particles. ${ }^{8,9,41}$ Therefore, developing a clearer understanding of the distribution or localization of a crosslinking agent in the PMMA matrix is a useful objective. To achieve this, latexes with deuterated PMMA cores were prepared to enable contrast-variation SANS measurements. These particles were sized using TEM and DLS to ensure that the deuteration of the core-forming monomer did not impact the particle structure. As can be seen in the TEM micrographs (Figure S1) and particle diameter distributions (Figure 3), this does not appear to be the case, with particles of a similar size and spherical morphology obtained for both MMA and MMA- $d_{8}$. Similarly, the sizes and distributions of crosslinked particles were very close to their noncrosslinked
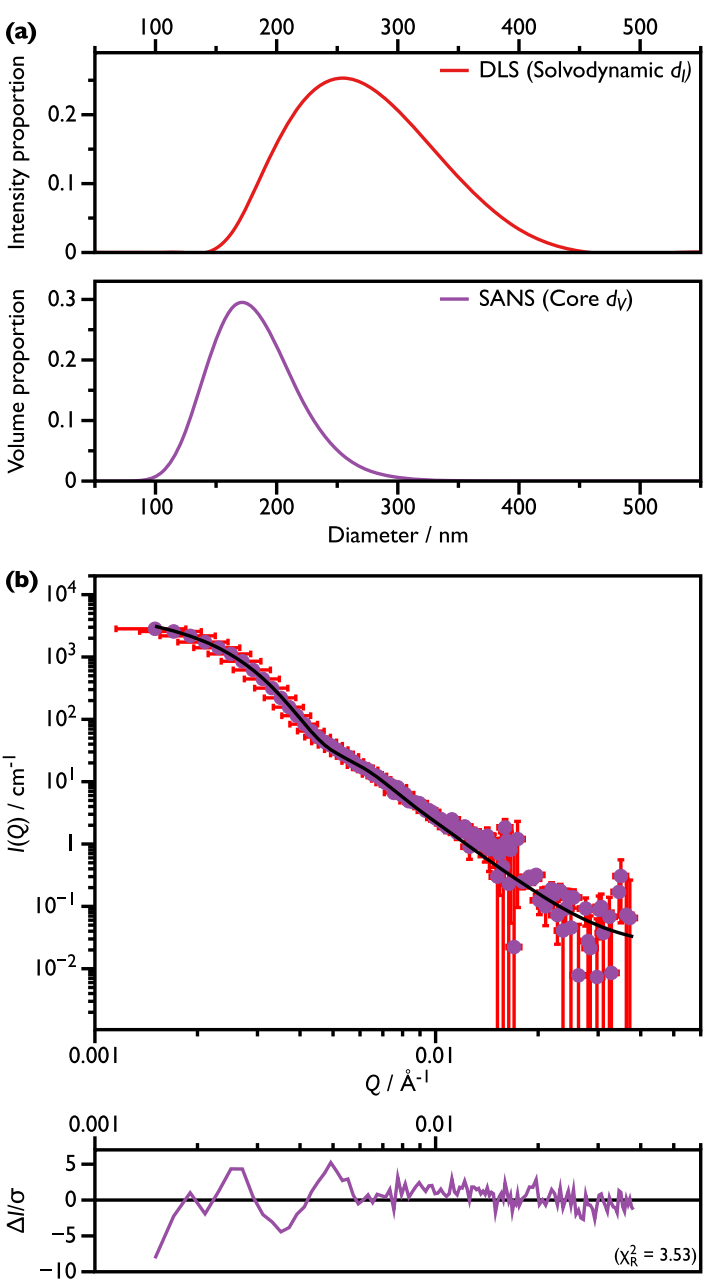

Figure 2. Size distributions (a) and SANS data (b) for $\mathrm{H}_{252}$. The size distributions are determined from SANS (volume-weighted) and DLS (intensity-weighted), and the SANS distribution is shifted to smaller sizes compared to DLS. This is because SANS is mostly sensitive to the particle core, given the minimal contrast between the solvent and stabilizer shell in these measurements (Table 2), whereas DLS measures the solvodynamic size and so is sensitive the stabilizer as well. The dilute SANS data in (b) were fitted using a spherical form factor (eq 5, solid line) with fitting parameters given in Table S1.

counterparts. The particle diameters determined from DLS measurements and fits to the EM histograms are given in Table 3.

The benefit of using deuterated-core latexes in multiple solvent SLDs is that the distribution of components can be determined. As we are now using multiple, nonhydrogenous solvent compositions, the PDMS-stabilizer is no longer effectively contrast matched to the alkane solvent, so there will be a contribution to the scattering from stabilizer molecules covalently linked to the particle surface. To enable an informed fit to the data from the PDMS at the particle interface, the scattering from a solution of the PDMS-MA homopolymer was measured in dodecane- $d_{26}$ and was fit to a model for polymer chains subject to excluded volume effects (SasView polymerexcl_volume $\operatorname{model}^{66}$ ), originally presented by Benoit and later refined. ${ }^{67-70}$ We have used Hammouda's expression, ${ }^{68}$ where the form factor $(P(Q)$, eq 6 is calculated from the excluded volume parameter $(\nu$, which is the inverse of the Porod exponent $m)$ and the radius of gyration $\left(R_{\mathrm{g}}\right)$ via the term $U(U=$ 

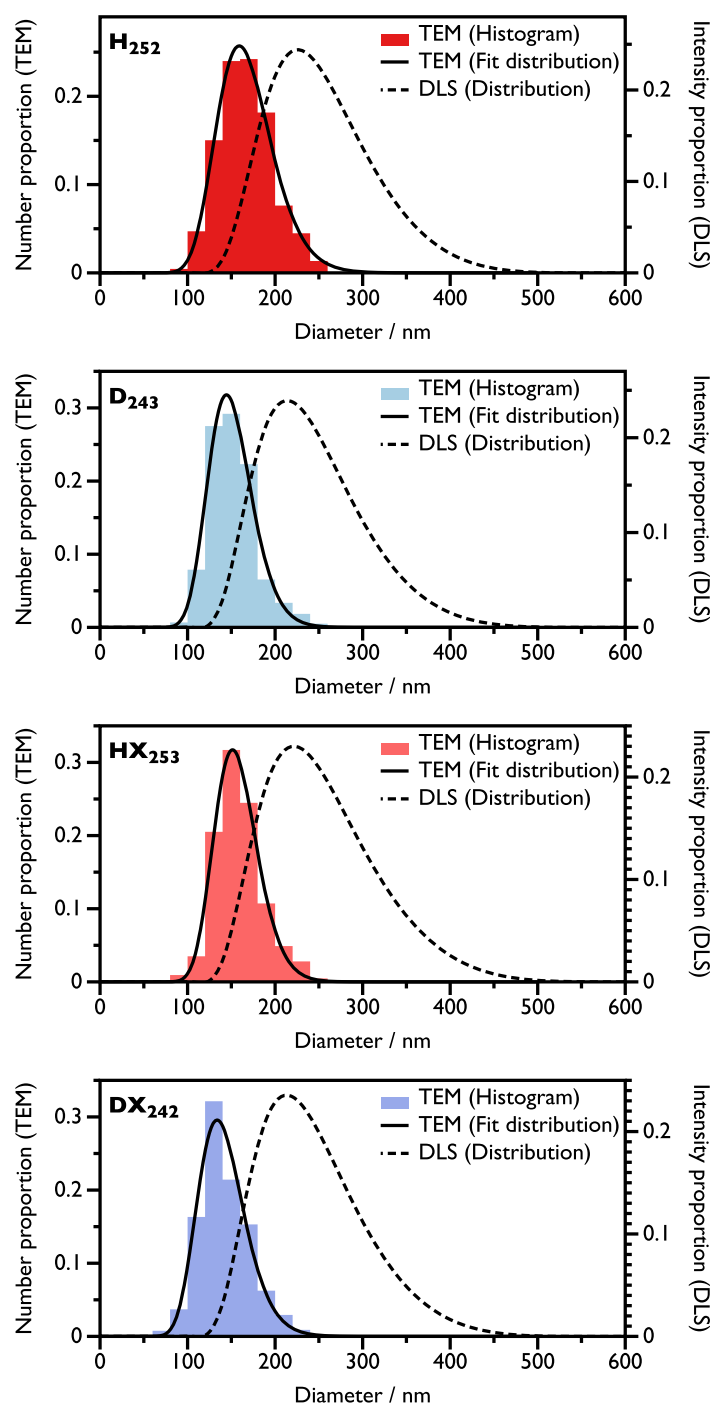

Figure 3. Particle size distributions obtained for PDMS-stabilized PMMA latexes to investigate the role of incorporating crosslinker or deuterating the PMMA core. The particle diameters are essentially the same for all particles, with only a slight broadening seen in the DLS size distributions for deuterated and crosslinked latexes.

$\left.\left(Q^{2} R_{\mathrm{g}}{ }^{2}(2 \nu+1)(2 \nu+2)\right) / 6\right) \cdot \gamma(x, U)$ is the incomplete gamma function, $\gamma(x, U)=\int_{0}^{U} \mathrm{~d} t \exp (-t) t^{x-1}$.

$$
P(Q)=\frac{1}{\nu U^{1 / 2 \nu}}\left\{\gamma\left(\frac{1}{2 \nu}, U\right)-\frac{1}{U^{1 / 2 \nu}} \gamma\left(\frac{1}{\nu}, U\right)\right\}
$$

The radius of gyration $\left(R_{\mathrm{g}}\right)$ for a Gaussian chain polymer coil can be calculated using eq 7. $L$ is the contour length, $b$ is the Kuhn length, and $L / b \equiv N$ is the number of Kuhn segments. ${ }^{71}$

$$
R_{\mathrm{g}}=\sqrt{\frac{L / b}{6}} \cdot b \equiv \sqrt{\frac{N}{6}} \cdot b
$$

This is a special case $(\nu=1 / 2)$ of a general expression (eq 8) to calculate $R_{\mathrm{g}}$ from polymers with any excluded volume parameter. ${ }^{66}$

$$
R_{\mathrm{g}}=\sqrt{\frac{b^{2}(L / b)^{2 \nu}}{(2 \nu+1)(2 \nu+2)}}
$$

The contour length of the polymers $(L)$ can be calculated as the product of the degree of polymerization and the projected contour length of each unit $(\mathrm{O}-\mathrm{Si}-\mathrm{O}$ unit in an all-trans configuration, $3.18 \AA$ ); values are taken from hexamethyldisiloxane in the gas phase. ${ }^{72}$ The Kuhn length $(b)$ of PDMS in the melt is $15.6 \AA \mathrm{A}^{.3}$ From these values, the $R_{\mathrm{g}}$ of the PDMS used here is estimated to be $52 \AA$.

SANS data were measured from a dilute solution of the unreacted PDMS and is shown in Figure 4 and fit using the

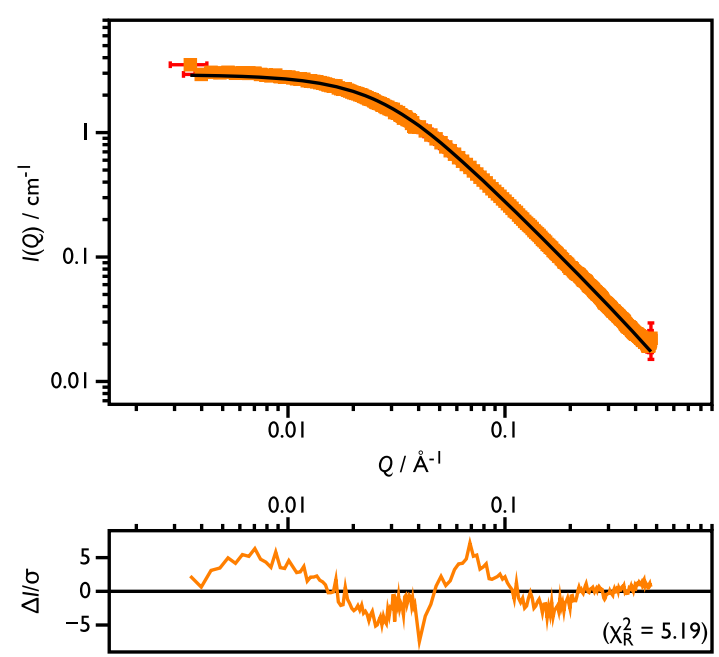

Figure 4. SANS data obtained for PDMS solution in dodecane- $d_{26}$. The solid line is the best fit to the data using a model for polymer chains subject to excluded volume effects. The best fit $R_{\mathrm{g}}$ is $51 \AA$ and for $\nu$ is 0.57 . The error-weighted difference function $(\Delta I / \sigma)$ is shown below the scattering data.

model described above. In this model, the excluded volume parameter $\nu$ is not assumed to take the value for the pure Gaussian model $(\nu=1 / 2)$ and instead is applicable for $\nu$ in the mass fractal range $(1 / 3 \leq \nu \leq 3 / 5)$. Swollen polymers in good solvents $(\nu=3 / 5)$ have values greater than $1 / 2$, and precipitated polymers in bad solvents $(\nu=1 / 3)$ have values less than $1 / 2$. The best fit value of $\nu$ is 0.57 , which shows that this PDMS is slightly swollen in the solvent. The best fit value of the $R_{\mathrm{g}}$ from SANS data is $51 \AA$, which compares favorably to the value calculated using a Gaussian coil $(52 \AA)$ but is less than calculated for the fit $\nu$ parameter $(67 \AA)$.

The SANS data for the deuterated latexes were fit as a sum of three parameters to describe the scattering over the entire $Q$ range measured. At low- $Q$ scattering from the cores of the latexes dominates, and these data were fit using an "onion" shell model (SasView Onion model ${ }^{74}$ ), which consists of concentric layers with either a constant or varying (linearly or exponentially) scattering length density. The radius of the core is set to a small value ( $1 \AA$ ), so the actual core of the latexes is represented by layers with varying scattering length densities. The form factor $(P(Q))$ of such a sphere is defined as the square of the form factor amplitudes $(f(Q))$ normalized by the particle volume $\left(V_{\mathrm{p}}\right.$, the volume of the sphere where the scattering length density is not equal to that of the solvent).

$$
P(Q)=[f(Q)]^{2} / V_{\mathrm{p}}
$$

The form factor amplitudes for the core $\left(f_{c}\right.$, eq 10$)$, the layers $\left(f_{\mathrm{l}}\right.$, eq 11$)$, and the solvent medium $\left(f_{\mathrm{m}}\right.$, eq 12$)$ are given below, where $r$ is the radii of the layers $\left(r_{\mathrm{c}}\right.$ for the core radius, $r_{1}$ for the layer thickness, and $r_{\mathrm{N}}$ for the entire particle) with corresponding scattering length densities $(\rho)$. $j_{1}(x)$ is the first- 
order spherical Bessel function $\left(j_{l}(x)=\left((\sin (x)-x \cdot \cos x) / x^{2}\right)\right.$, and the volume $(V(r))$ is $((4 \pi) / 3) r^{3}$.

$$
\begin{aligned}
& f_{\mathrm{c}}(Q)=4 \pi \int_{0}^{r_{\mathrm{c}}} \rho_{\mathrm{c}} \frac{\sin (Q r)}{Q r} r^{2} \mathrm{~d} r=3 \rho_{\mathrm{c}} V\left(r_{\mathrm{c}}\right) \frac{j_{1}\left(Q r_{\mathrm{c}}\right)}{Q r_{\mathrm{c}}} \\
& f_{\mathrm{l}}(Q)=4 \pi \int_{r_{\mathrm{l}}-1}^{r_{1}} \rho_{1}(r) \frac{\sin (Q r)}{Q r} r^{2} \mathrm{~d} r \\
& f_{\mathrm{m}}(Q)=4 \pi \int_{r_{\mathrm{N}}}^{\infty} \rho_{\mathrm{m}} \frac{\sin (Q r)}{Q r} r^{2} \mathrm{~d} r=-3 \rho_{\mathrm{m}} V\left(r_{\mathrm{N}}\right) \frac{j_{1}\left(Q r_{\mathrm{N}}\right)}{Q r_{\mathrm{N}}}
\end{aligned}
$$

Further details about the model, and example calculations of the SLD profiles and form factor amplitudes are provided in the SasView documentation. ${ }^{74}$ Unlike the $\mathrm{H}_{252}$ spheres, where the stabilizer could be ignored, the contrast between the hydrogenous PDMS and mostly deuterated solvent means that it is a significant component and cannot be disregarded.

At middle $Q$ the scattering from the PDMS chains themselves dominate, and these were fit using the polymer chains subject to excluded volume effects model, discussed earlier. At high $Q$ a constant background dominates, arising from the incoherent and instrumental backgrounds.

The SANS data for the noncrosslinked $\mathrm{D}_{243}$ latexes in two solvents with different SLDs (shown in the legend) are shown in Figure 5. These two solvents were chosen to provide contrast

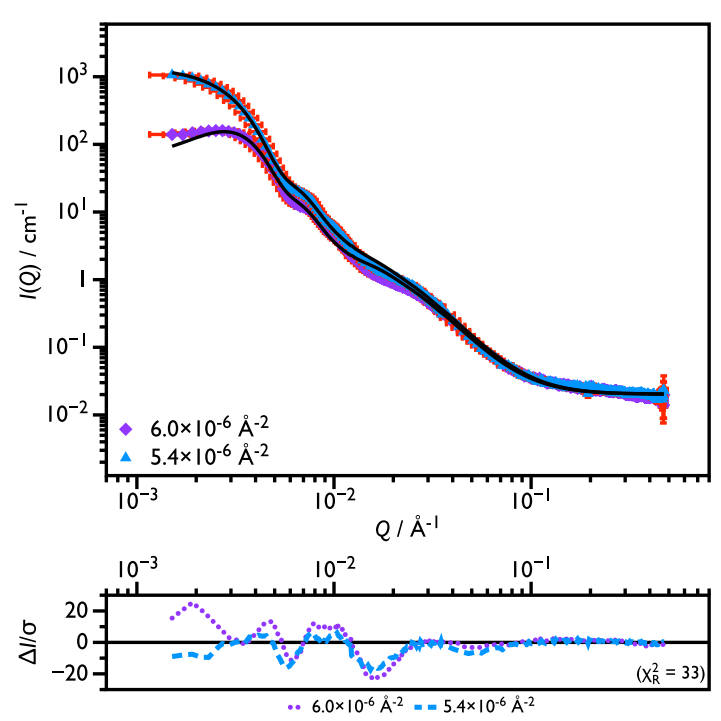

Figure 5. SANS obtained for noncrosslinked PMMA- $d_{8}$ PDMSstabilized latexes $\left(D_{243}\right)$. Three-component fits to the experimental SANS data are shown, and good agreement is achieved over the whole $Q$ range (fitting parameters given in Tables $S 2$ and S3), as shown in the error-weighted difference function $(\Delta I / Q)$.

with the PMMA- $d_{8}$ core, which has an SLD greater than dodecane- $d_{26}$ (Table 2 ). The "onion" shell model is necessary to fit this data, which can be seen by the shapes of the scattering data in Figure 5. The curves would be the same shape if the scattering originated from a homogeneous structure, and an attempt at fitting the data with a sphere model (as was the case for $\mathrm{H}_{252}$ in dodecane) was unsuccessful (Figure S2). The data in different solvents were simultaneously fit with all parameters either set to known values or simultaneously fit, other than the background, the scale of the polymer scattering, and the SLD at the core-shell interface. The data were fit in two steps: first, models for the interfacial PDMS and the background were fitted in the range where their contribution is the dominant component $\left(Q>0.02 \AA^{-1}\right)$ of the scattering intensity, and then the model for the latex was fitted to the residual scattering. The three components that contribute to the total scattering curve are shown in Figure 6. A good fit is achieved for both
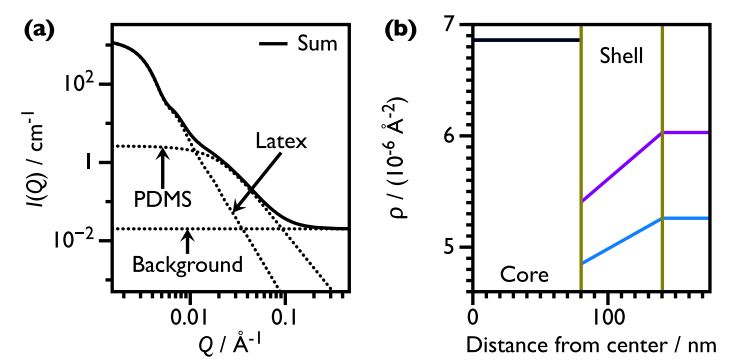

Figure 6. Linear combination of components is shown (a) for the data from $\mathrm{D}_{243}$ latexes in dodecane with an SLD, $\rho=6.0 \times 10^{-6} \AA^{-2}$. The SLD-distance profile is shown (b) for the latex scattering model, which shows that the best description the data is a core with a constant SLD equal to that of pure PMMA- $d_{8}$ (Table 2 ) and a linearly varying SLD in the shell (colors in the shell and solvent correspond to data in Figure 5).

solvent contrasts for both particles, especially considering that so many parameters are constrained between the two. The fits to the data provide information about the structure of both the core and shell of the particles.

The scattering from the PDMS polymer is not identical to that in solution. The value of $R_{\mathrm{g}}$ has increased ( $88 \AA$ ), and the value of $\nu$ was fixed to the limit of applicability for the model $(\nu=1 / 3)$, as it would otherwise vary to nonphysical values. This suggests that the distribution of PDMS at the interface is as if it were collapsed, compared to the solution. This initially is surprising; PDMS is an effective steric stabilizer for these latexes. The polymer, however, is a dimethacrylate, and it will likely react at both ends to form loops at the surface. This nonrandom distribution of the polymer appears in the scattering data as a collapsed polymer. This does not mean that we believe the PDMS to have collapsed and to be an ineffective steric stabilizer. Rather, it is due to the spatial distribution of a polymer that is reactive at both ends compared to a free polymer in solution. The $R_{\mathrm{g}}$ is much greater than would be expected given this value of $\nu(30 \AA)$, which suggests that the polymer is not equivalent to one free in solution. This emphasizes that the scattering only is consistent with a polymer with these fit values of $R_{\mathrm{g}}$ and $\nu$, not that the PDMS polymer itself is distributed as such. While this is a consistent explanation, it is worth noting that the scattering from this contribution is obscured by the latex scattering at low $Q$ and the flat background at high $Q$ and it, therefore, is hard to resolve with complete certainty.

A difference in SLD between the core and the shell is necessary to fit the data. These cannot be successfully fit if they are considered to be homogeneous spheres, as was the case for the hydrogenous $\mathrm{H}_{252}$ latex (Figure 2). This demonstrates the incredible sensitivity of neutron scattering for small differences in composition. The fit SLDs of the shells represent $\sim 5 \%$ by volume of PDMS in the shell, but this small proportion of the polymer in the shell is clearly detectable by SANS. This is comparable to the amount of the solvent in the shell for PHSAstabilized latexes. ${ }^{19,75}$

Geometrically, the exponential variation of the SLD across the shell must be a decay, and the best fit to the data is achieved in 

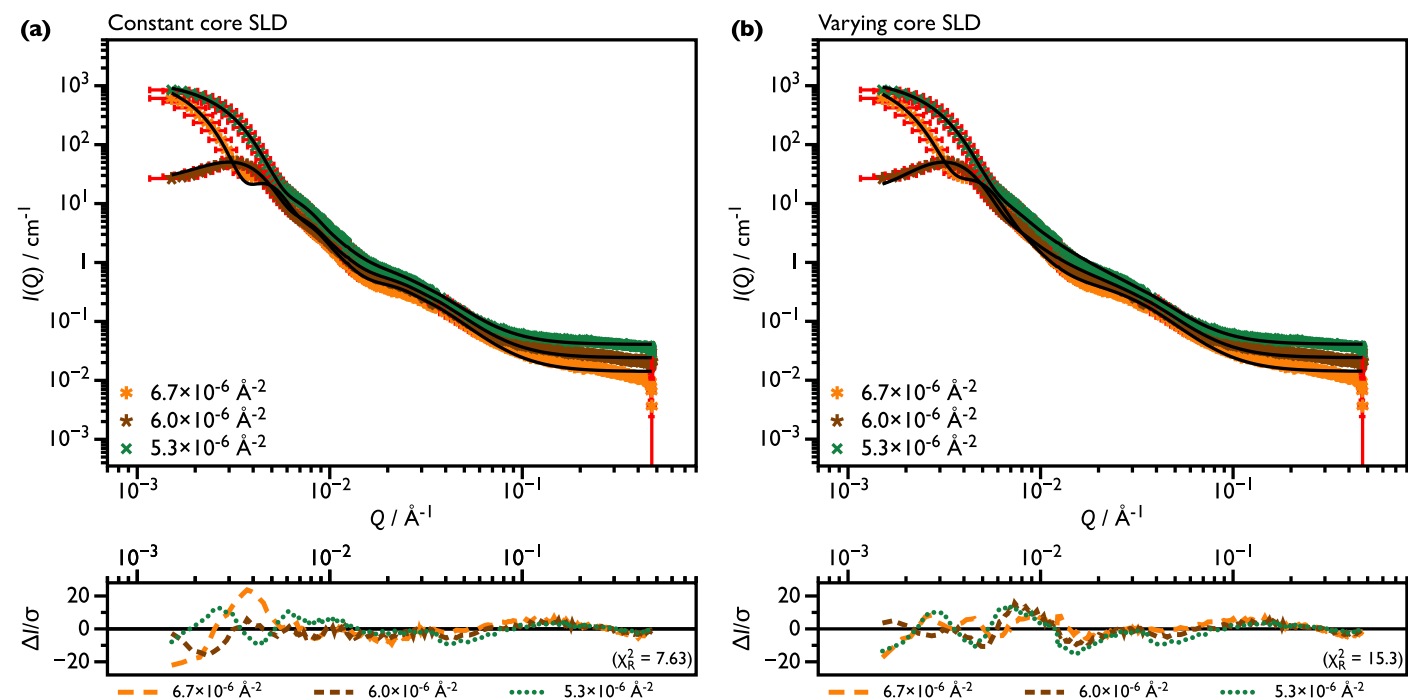

Figure 7. SANS obtained for crosslinked PMMA- $d_{8}$ PDMS-stabilized latexes $\left(\mathrm{DX}_{242}\right)$. As for the data in Figure 5, three-component fits to the experimental SANS data are shown along with the error-weighted difference function $(\Delta I / Q)$. Two different core SLD profiles $[$ a constant SLD (a) and an exponentially varying SLD (b)] are used to fit the data, and they give similarly good fits to the data. Fitting parameters are given in Tables S4 and S5 and S4 and S6 respectively.

the limit where this is a linear variation. We find that the fit thickness of the stabilizer layer is much larger than reported for PHSA branch-stabilizer (typically $10 \mathrm{~nm})^{3,16,75}$ and persists for approximately $60 \mathrm{~nm}$ from the particle core into the solvent. This supports the earlier hypothesis (from DLS and EM) that the solvodynamic diameter is larger than the core diameter due to the stabilizer layer significantly increasing the effective particle size. Given that the fully elongated contour length of the PDMS polymer is $106 \mathrm{~nm}$, this shell thickness is physically realistic.

Finally, we note that the SLD of the core was fixed to the value for pure PMMA- $d_{8}$ homopolymer (Table 2 ). There was no need to vary it to achieve a good fit to the data, and in fact, the value of the SLD did not change when the parameter was allowed to float. This suggests that there is strong segregation between the PDMS stabilizer and the PMMA core and that the PMMA is similar to the homopolymer, which contrasts with other PMMA particles that were found to have lower core densities or a microphase structure. ${ }^{19,76-78}$

The same type of SANS data was also obtained from the crosslinked $\mathrm{DX}_{242}$ latexes as well, and the data were treated in the same way. As for the $\mathrm{D}_{243}$ latexes, an attempt to fit the data with a homogeneous sphere model was unsuccessful (Figure S3). In this case, the scattering in a third solvent (pure dodecane- $d_{26}$ ) was measured as well, as its $\operatorname{SLD}\left(6.71 \times 10^{-6} \AA^{-2}\right)$ will be commensurate to or greater than the SLD of the core of the crosslinked latex. This means that there are now solvents with SLDs both greater than and less than the latexes, which will enable us to somewhat overcome the phase problem. ${ }^{79}$ The data for the crosslinked $\mathrm{DX}_{242}$ latexes are shown in Figure 7 .

In general, the data for the crosslinked latexes can be fit the same as for the noncrosslinked latexes using a three-component model. However, the SLD of the cores of the latexes are different. This should be expected given that the crosslinked latexes have an additional hydrogenous component (EGDMA crosslinker), but the fits to the data reveal an interesting detail about the distribution of the crosslinker.

It was not possible to fit the $\mathrm{DX}_{242}$ data if the core SLD was set to that of PMMA- $d_{8}$ using any set of parameters. Good fits to the data can be obtained using two different SLD distributions in the core of the latexes (shown in Figure 8). We fit the data using two assumed distributions of the scattering length density in the core

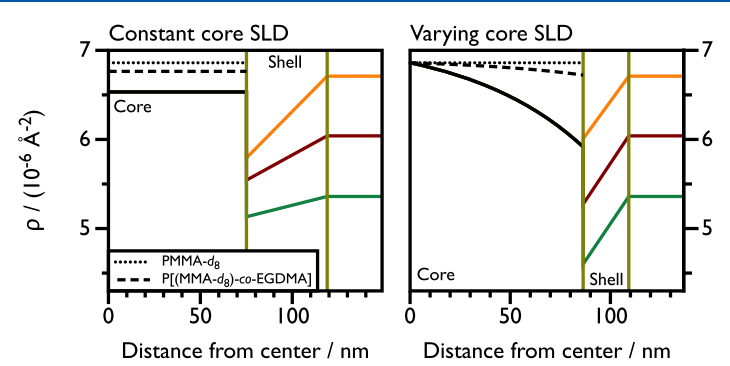

Figure 8. SLD-distance profiles for fits to the SANS data shown in Figure 7 for two possible distributions of hydrogenous material, constant core SLD (left) and exponentially varying core SLD (right). The best fit for the shell is with a linearly varying SLD in the shell (colors in the shell and solvent correspond to data in Figure 7). The best fit for the core, using either model, gives an SLD (solid line) that is lower than either PMMA- $d_{8}$ (dotted line) or calculated for the crosslinked copolymer $\mathrm{P}\left[\left(\mathrm{MMA}-d_{8}\right)\right.$-co-EGDMA $]$ known from sample preparation (Table 1) (dashed line).

of the latexes: either a constant SLD (hydrogenous material uniformly distributed) or an exponentially varying SLD (hydrogenous material preferentially distributed near the center or the interface). We can achieve similarly good fits to the data using both of these models. For the constant core SLD case, the fit minimization is better (lower $\chi_{\mathrm{R}}{ }^{2}$ ), but for the varying core $\mathrm{SLD}$, the error-weighted difference function is lower across the whole $Q$ range. Therefore, from the quality of the fits alone, we are unable to prefer one description over the other. Both give consistent descriptions of the distribution of hydrogenous molecules in the core of the latexes, though, so the distinction may not be crucial. As can be seen by comparing the hypothetical values of the core SLDs calculated if the core consisted only of the $\mathrm{P}\left[\left(\mathrm{MMA}-d_{8}\right)\right.$-co-EGDMA $]$ copolymer to the fit values, the real SLD is significantly lower than the hypothetical value. Beyond the EGDMA crosslinker, the only other hydrogenous species in the system is the PDMS stabilizer. 
These data suggest that the PDMS is not strongly segregated to the interface and, rather, seems to be distributed throughout the core of the latexes as well. Using the fit SLD values, we calculate that, if the PDMS stabilizer were the only other hydrogenous species in the system, a few percent by volume PDMS in the core ( $3 \%$ for constant core or $7 \%$ for varying core) would be sufficient to lower the SLD to match the fit values. This calculation suggests some preference for the varying core model over the constant core model. If we calculate the combined amount of PDMS in the core and in the shell as a proportion of the total amount of polymer for the constant core case, we find that it varies as a function of solvent SLD. However, if we repeat the same calculation for the varying core case, we find that it is essentially the same. The amount of PDMS compared to MMA and EGDMA monomers from this calculation is less than that predicted from the recipe (Table 1), but this could be due to the loss of unreacted and soluble PDMS. Again, this highlights the sensitivity of neutron scattering for detecting small differences in composition.

We suggest that this could be caused by the reduced mobility of the polymer chains upon adding EGDMA. Crosslinking the core will constrain the motion of the polymer chains. The PDMS stabilizer is introduced during the synthesis step, and it will partition to the interface because of a preference for the solvent over PMMA. However, if the mobility of the PMMA chains is reduced, some PDMS may be entrapped in the core of the particle, reducing the core SLD. This demonstrates the additional compositional information that can be obtained using SANS for otherwise "model" colloids. The noncrosslinked PMMA- $d_{8}$ latexes seem to be more "model" than other PMMA colloids produced with other stabilizers. ${ }^{19,76-78}$ However, the crosslinked particles, which include a functional monomer to introduce desirable stability, do not seem to have such a uniform internal composition. They may be model colloids from a physical perspective, but they are nonetheless complex chemically.

\section{CONCLUSIONS}

In this work, we have studied the structure of PDMS-stabilized PMMA nanoparticles. By producing particles spanning an order of magnitude in size, we are able to study particle properties over a range of length scales. In this way, we extend earlier work to push the size of PMMA particles to smaller sizes than previously obtained $^{33,34}$ for grafted PDMS stabilizers, accessing the size regime that can be studied using small-angle scattering methods. The particle size distribution of these small particles is greater than for the equivalent particles produced using the PHSA stabilizer, although there are still sufficient features in the scattering data to obtain good quality fits. Moreover, PDMS stabilizers provide a greater degree of scattering contrast to the PMMA particle core compared to PHSA stabilizer, allowing for a clearer form factor determination than previously obtained. It is also important to note that in this instance the PDMS stabilizer enhances the particle diameter by more than the $\sim 20$ $\mathrm{nm}$ detected for PHSA stabilizers. This raises some questions as to the suitability of PDMS-stabilized PMMA particles in experiments that require "model hard spheres", but they can be successfully used in applications where a softer or more longranged repulsion is desirable. For example, because of the wide range of molecular weights that can be readily obtained for PDMS-MA, this raises the intriguing possibility of producing PMMA particles with a much finer control of "hardness" compared to their PHSA analogues, with exciting application in the study of colloidal phase behavior in multicomponent mixtures. $^{27,80}$ We also investigate the role of EGDMA in the particle structure. EGDMA, typically added dropwise to the PMMA synthesis as a crosslinking agent, ${ }^{8,9}$ appears to be located more-or-less homogeneously throughout the core of the particle, as previously thought, ${ }^{42}$ at least within the ability of SANS measurements to resolve it. However, the SANS measurements do show that the EGDMA does not appear to be the only nonPMMA species in the core of the latexes, and we suggest that the PDMS stabilizer is also trapped within the core of the latex. Future work will address this question by using PDMS stabilizers of different molecular weights and with monomethacryloxypropyl termination, which should have different mobility in the polymerizing PMMA matrix, enhancing or suppressing the trapping of PDMS in the particle core.

\section{ASSOCIATED CONTENT}

\section{Supporting Information}

The Supporting Information is available free of charge at https://pubs.acs.org/doi/10.1021/acs.langmuir.9b03911.

Electron micrographs and further details on fitting scattering data (PDF)

\section{AUTHOR INFORMATION}

\section{Corresponding Author}

James E. Hallett - H. H. Wills Physics Laboratory, University of Bristol, Bristol BS8 1TL, U.K.; 10 orcid.org/0000-0002-97479980; Email: james.hallett@chem.ox.ac.uk

\section{Authors}

Isabelle Grillo - Institut Laue-Langevin, 38042 Grenoble Cedex 9, France

Gregory N. Smith - Department of Chemistry, University of Sheffield, Sheffield, South Yorkshire S3 7HF, U.K.; Niels Bohr Institute, H. C. Ørsted Institute, University of Copenhagen, 2100 Copenhagen, Denmark; 이이.org/0000-0002-0074-5657

Complete contact information is available at:

https://pubs.acs.org/10.1021/acs.langmuir.9b03911

\section{Notes}

The authors declare no competing financial interest.

\#(I.G.) Deceased (5 August 2019).

\section{ACKNOWLEDGMENTS}

The Institut Laue-Langevin (experiment 9-12-478, raw data available from doi.org/10.5291/ILL-DATA.9-12-478) is acknowledged for allocation of beamtime, ${ }^{81}$ and the UK Science and Technology Facilities Council for funding travel. Dr Jocelyn Peach and Dr Jonathan Pegg are thanked for performing preliminary measurements. G.N.S. acknowledges Professor S. P. Armes (University of Sheffield, UK) for support and funding from the ERC via a five-year Advanced Investigator grant (PISA 320372) and EPSRC (EP/J007846) while working in his group. J.E.H. acknowledges Professor C. P. Royall (University of Bristol, UK) for support and funding from the ERC via the NANOPRS consolidator grant, project 617266 while working in his group. The authors acknowledge Professor T. Cosgrove (University of Bristol, UK) for valuable technical discussions. The open access fee was covered by FILL2030, a European Union project within the European Commission's Horizon 2020 Research and Innovation programme under grant 
agreement $\mathrm{N}^{\circ} 731096$. This article is dedicated to the memory of Isabelle Grillo.

\section{REFERENCES}

(1) Cairns, R. J. R.; Ottewill, R.; Osmond, D.; Wagstraff, I. Studies on the preparation and properties of latices in nonpolar media. J. Colloid Interface Sci. 1976, 54, 45-51.

(2) Antl, L.; Goodwin, J. W.; Hill, R. D.; Ottewill, R. H.; Owens, S. M.; Papworth, S.; Waters, J. A. The preparation of poly (methyl methacrylate) latices in non-aqueous media. Colloids Surf. 1986, 17, 67-78.

(3) Cebula, D. J.; Goodwin, J. W.; Ottewill, R. H.; Jenkin, G.; Tabony, J. Small angle and quasi-elastic neutron scattering studies on polymethylmethacrylate latices in nonpolar media. Colloid Polym. Sci. 1983, 261, 555-564.

(4) Royall, C. P.; Poon, W. C. K.; Weeks, E. R. In search of colloidal hard spheres. Soft Matter 2013, 9, 17-27.

(5) Hsu, M. F.; Dufresne, E. R.; Weitz, D. A. Charge Stabilization in Nonpolar Solvents. Langmuir 2005, 21, 4881-4887.

(6) Sánchez, R.; Bartlett, P. Synthesis of charged particles in an ultralow dielectric solvent. Soft Matter 2011, 7, 887-890.

(7) Smith, G. N.; Hallett, J. E.; Eastoe, J. Celebrating Soft Matter's 10th Anniversary: Influencing the charge of poly (methyl methacrylate) latexes in nonpolar solvents. Soft Matter 2015, 11, 8029-8041.

(8) Dullens, R. P. A.; Claesson, M.; Derks, D.; Van Blaaderen, A.; Kegel, W. K. Monodisperse core-shell poly (methyl methacrylate) latex colloids. Langmuir 2003, 19, 5963-5966.

(9) Elsesser, M. T.; Hollingsworth, A. D.; Edmond, K. V.; Pine, D. J. Large Core- Shell Poly (methyl methacrylate) Colloidal Clusters: Synthesis, Characterization, and Tracking. Langmuir 2011, 27, 917927.

(10) Gillespie, D. A. J.; Hallett, J. E.; Elujoba, O.; Che Hamzah, A. F.; Richardson, R. M.; Bartlett, P. Counterion condensation on spheres in the salt-free limit. Soft Matter 2014, 10, 566-577.

(11) Hussain, G.; Robinson, A.; Bartlett, P. Charge generation in lowpolarity solvents: Poly (ionic liquid)-functionalized particles. Langmuir 2013, 29, 4204-4213.

(12) Hallett, J. E.; Gillespie, D. A. J.; Richardson, R. M.; Bartlett, P. Charge regulation of nonpolar colloids. Soft Matter 2018, 14, 331-343.

(13) Bartlett, P.; Ottewill, R. H. Geometric interactions in binary colloidal dispersions. Langmuir 1992, 8, 1919-1925.

(14) Bartlett, P.; Ottewill, R. H. A neutron scattering study of the structure of a bimodal colloidal crystal. J. Chem. Phys. 1992, 96, 33063318.

(15) Livsey, I.; Ottewill, R. H. A light scattering study of concentrated dispersions in nonaqueous media. Colloid Polym. Sci. 1989, 267, 421428.

(16) Markovic, I.; Ottewill, R. H.; Underwood, S. M.; Tadros, T. F. Interactions in concentrated nonaqueous polymer latices. Langmuir 1986, 2, 625-630.

(17) Kemp, R.; Sanchez, R.; Mutch, K. J.; Bartlett, P. Nanoparticle charge control in nonpolar liquids: Insights from small-angle neutron scattering and microelectrophoresis. Langmuir 2010, 26, 6967-6976.

(18) Smith, G. N.; Alexander, S.; Brown, P.; Gillespie, D. A. J.; Grillo, I.; Heenan, R. K.; James, C.; Kemp, R.; Rogers, S. E.; Eastoe, J. Interaction between surfactants and colloidal latexes in nonpolar solvents studied using contrast-variation small-angle neutron scattering. Langmuir 2014, 30, 3422-3431.

(19) Smith, G. N.; Finlayson, S. D.; Gillespie, D. A. J.; Peach, J.; Pegg, J. C.; Rogers, S. E.; Shebanova, O.; Terry, A. E.; Armes, S. P.; Bartlett, P.; Eastoe, J. The internal structure of poly(methyl methacrylate) latexes in nonpolar solvents. J. Colloid Interface Sci. 2016, 479, 234-243.

(20) Smith, G. N.; Ahualli, S.; Delgado, Á. V.; Gillespie, D. A. J.; Kemp, R.; Peach, J.; Pegg, J. C.; Rogers, S. E.; Shebanova, O.; Smith, N.; Eastoe, J. Charging poly (methyl methacrylate) latexes in nonpolar solvents: Effect of particle concentration. Langmuir 2017, 33, 1354313553.

(21) van der Linden, M. N.; Stiefelhagen, J. C. P.; Heessels-Gürboğa, G.; van der Hoeven, J. E. S.; Elbers, N. A.; Dijkstra, M.; van Blaaderen,
A. Charging of poly (methyl methacrylate)(PMMA) colloids in cyclohexyl bromide: Locking, size dependence, and particle mixtures. Langmuir 2014, 31, 65-75.

(22) Vissers, T.; van Blaaderen, A.; Imhof, A. Band formation in mixtures of oppositely charged colloids driven by an ac electric field. Phys. Rev. Lett. 2011, 106, 228303.

(23) Vissers, T.; Wysocki, A.; Rex, M.; Löwen, H.; Royall, C. P.; Imhof, A.; van Blaaderen, A. Lane formation in driven mixtures of oppositely charged colloids. Soft Matter 2011, 7, 2352-2356.

(24) Bartlett, P.; Campbell, A. I. Three-dimensional binary superlattices of oppositely charged colloids. Phys. Rev. Lett. 2005, 95, 128302.

(25) Leunissen, M. E.; Christova, C. G.; Hynninen, A.-P.; Royall, C. P.; Campbell, A. I.; Imhof, A.; Dijkstra, M.; Van Roij, R.; Van Blaaderen, A. Ionic colloidal crystals of oppositely charged particles. Nature 2005, $437,235-240$.

(26) Filion, L.; Hermes, M.; Ni, R.; Vermolen, E.; Kuijk, A.; Christova, C.; Stiefelhagen, J.; Vissers, T.; Van Blaaderen, A.; Dijkstra, M. Selfassembly of a colloidal interstitial solid with tunable sublattice doping. Phys. Rev. Lett. 2011, 107, 168302.

(27) Ríos de Anda, I.; Turci, F.; Sear, R. P.; Royall, C. P. Long-lived non-equilibrium interstitial solid solutions in binary mixtures. J. Chem. Phys. 2017, 147, 124504.

(28) Taffs, J.; Williams, S. R.; Tanaka, H.; Royall, C. P. Structure and kinetics in the freezing of nearly hard spheres. Soft Matter 2013, 9, $297-$ 305.

(29) Elsesser, M. T.; Hollingsworth, A. D. Revisiting the synthesis of a well-known comb-graft copolymer stabilizer and its application to the dispersion polymerization of poly(methyl methacrylate) in organic media. Langmuir 2010, 26, 17989-17996.

(30) Croucher, M. D.; Milkie, T. H. Temperature dependence of the shear viscosity of sterically stabilised polymer colloids. Faraday Discuss. Chem. Soc. 1983, 76, 261-276.

(31) Pelton, R. H.; Osterroth, A.; Brook, M. A. Silicone stabilized poly (methyl methacrylate) nonaqueous latexes: I. Preparation and characterization. J. Colloid Interface Sci. 1990, 137, 120-127.

(32) Pelton, R.; Osterroth, A.; Brook, M. A. Silicone stabilized poly (methyl methacrylate) nonaqueous latex: 2. Flocculation by degradation of the steric layer. J. Colloid Interface Sci. 1991, 147, 523-530.

(33) Klein, S. M.; Manoharan, V. N.; Pine, D. J.; Lange, F. F. Preparation of monodisperse PMMA microspheres in nonpolar solvents by dispersion polymerization with a macromonomeric stabilizer. Colloid Polym. Sci. 2003, 282, 7-13.

(34) Richez, A. P.; Farrand, L.; Goulding, M.; Wilson, J. H.; Lawson, S.; Biggs, S.; Cayre, O. J. Dispersion polymerization of poly(dimethylsiloxane)-stabilized polymer particles from radical dispersion polymerization in nonpolar solvent: Influence of stabilizer properties and monomer type. Langmuir 2014, 30, 1220-1228.

(35) Kodger, T. E.; Lu, P. J.; Wiseman, G. R.; Weitz, D. A. Stable, fluorescent polymethylmethacrylate particles for the long-term observation of slow colloidal dynamics. Langmuir 2017, 33, 63826389.

(36) Choi, G. N.; Krieger, I. M. Rheological studies on sterically stabilized model dispersions of uniform colloidal spheres. II. Steadyshear viscosity. J. Colloid Interface Sci. 1986, 113, 101-113.

(37) Chop, G. N.; Krieger, I. M. Rheological studies on sterically stabilized dispersions of uniform colloidal spheres. I. Sample Preparation. J. Colloid Interface Sci. 1986, 113, 94-100.

(38) Keville, K. M.; Franses, E. I.; Caruthers, J. M. Preparation and characterization of monodisperse polymer microspheroids. J. Colloid Interface Sci. 1991, 144, 103-126.

(39) Lin, T.; Kodger, T. E.; Weitz, D. A. Transport of charged colloids in a nonpolar solvent. Soft Matter 2013, 9, 5173.

(40) Tsurusawa, H.; Russo, J.; Leocmach, M.; Tanaka, H. Formation of porous crystals via viscoelastic phase separation. Nat. Mater. 2017, $16,1022-1028$

(41) Pathmamanoharan, C.; Groot, K.; Dhont, J. K. G. Preparation and characterization of crosslinked PMMA latex particles stabilized by grafted copolymer. Colloid Polym. Sci. 1997, 275, 897-901. 
(42) Peng, B.; van der Wee, E.; Imhof, A.; van Blaaderen, A. Synthesis of Monodisperse, Highly Cross-Linked, Fluorescent PMMA Particles by Dispersion Polymerization. Langmuir 2012, 28, 6776-6785.

(43) Einstein, A. Eine neue Bestimmung der Moleküldimensionen. Ann. Phys. 1906, 324, 289-306.

(44) Einstein, A. Berichtigung zu meiner Arbeit: „Eine neue Bestimmung der Moleküldimensione”. Ann. Phys. 1911, 339, 591-592.

(45) CRC Handbook of Chemistry and Physics, 95th ed.; CRC Press, 2014-2015, (Internet Version).

(46) Fielding, L. A.; Derry, M. J.; Ladmiral, V.; Rosselgong, J.; Rodrigues, A. M.; Ratcliffe, L. P. D.; Sugihara, S.; Armes, S. P. RAFT dispersion polymerization in non-polar solvents: facile production of block copolymer spheres, worms and vesicles in $n$-alkanes. Chem. Sci. 2013, 4, 2081-2087.

(47) Schneider, C. A.; Rasband, W. S.; Eliceiri, K. W. NIH Image to ImageJ: 25 years of image analysis. Nat. Methods 2012, 9, 671-675.

(48) Doucet, M.; et al. SasView, version 4.2.2; Zenodo, 2019.

(49) Trewhella, J.; et al. 2017 publication guidelines for structural modelling of small-angle scattering data from biomolecules in solution: an update. Acta Crystallogr., Sect. D: Struct. Biol. 2017, 73, 710-728.

(50) Gall, W. G.; McCrum, N. G. Internal friction in stereoregular polymethyl methacrylate. J. Polym. Sci. 1961, 50, 489-495.

(51) Kataoka, T.; Ueda, S. Viscosity-molecular weight relationship for polydimethylsiloxane. J. Polym. Sci., Part B: Polym. Lett. 1966, 4, 317-322.

(52) Bridge, B.; Gabrys, B.; Joshi, S. B.; Higgins, J. S. The effect of deuteration on the ultrasonic properties of poly(methyl methacrylate). J. Mater. Sci. 1989, 24, 3295-3299.

(53) Sigma-Aldrich. http://www.sigmaaldrich.com/united-kingdom. html (accessed $1^{\text {st }}$ November 2019).

(54) Sears, V. F. Neutron scattering lengths and cross sections. Neutron News 1992, 3, 26-37.

(55) Winnik, M. A.; Lukas, R.; Chen, W. F.; Furlong, P. Studies of the dispersion polymerisation of methyl methacrylate in nonaqueous media. Makromol. Chem., Macromol. Symp. 1987, 10-11, 483-501.

(56) Kawaguchi, S.; Ito, K. Polymer Particles; Springer, 2005; pp 299328.

(57) Green, D. L.; Mewis, J. Connecting the wetting and rheological behaviors of poly (dimethylsiloxane)-grafted silica spheres in poly (dimethylsiloxane) melts. Langmuir 2006, 22, 9546-9553.

(58) Landherr, L. J. T.; Cohen, C.; Agarwal, P.; Archer, L. A. Interfacial friction and adhesion of polymer brushes. Langmuir 2011, 27, 9387-9395.

(59) Jones, E. R.; Mykhaylyk, O. O.; Semsarilar, M.; Boerakker, M.; Wyman, P.; Armes, S. P. How do spherical diblock copolymer nanoparticles grow during RAFT alcoholic dispersion polymerization? Macromolecules 2015, 49, 172-181.

(60) Docherty, P. J.; Derry, M. J.; Armes, S. P. RAFT dispersion polymerization of glycidyl methacrylate for the synthesis of epoxyfunctional block copolymer nanoparticles in mineral oil. Polym. Chem. 2019, 10, 603-611.

(61) Smith, G. N.; Mears, L. L.; Rogers, S. E.; Armes, S. P. Synthesis and electrokinetics of cationic spherical nanoparticles in salt-free nonpolar media. Chem. Sci. 2018, 9, 922.

(62) sphere-SasView 4.2.2 documentation. http://www.sasview. org/docs/user/models/sphere.html (accessed 20 $0^{\text {th }}$ January 2020).

(63) Rayleigh, L. The Incidence of Light upon a Transparent Sphere of Dimensions Comparable with the Wave-Length. Proc. R. Soc. London, Ser. A 1910, 84, 25-46.

(64) Guinier, A.; Fournet, G. Small-Angle Scattering of X-Rays; John Wiley \& Sons: New York, 1955.

(65) van Gruijthuijsen, K.; Bouwman, W. G.; Schurtenberger, P.; Stradner, A. Direct comparison of SESANS and SAXS to measure colloidal interactions. Europhys. Lett. 2014, 106, 28002.

(66) polymer_excl_volume-SasView 4.2.2 documentation. http:// www.sasview.org/docs/user/models/polymer \_excl \_volume.html (accessed $1^{\text {st }}$ November 2019).
(67) Benoit, H. La Diffusion de La Lumiere par des Macromolecules en Chaines en Solution dans un Bon Solvant. Comptes Rendus 1957, 245, 2244-2247.

(68) Hammouda, B. Polymer Characteristics; Springer: Berlin, Heidelberg, 1993; pp 87-133.

(69) Hore, M. J. A.; Hammouda, B.; Li, Y.; Cheng, H. CoNonsolvency of Poly(n-isopropylacrylamide) in Deuterated Water/ Ethanol Mixtures. Macromolecules 2013, 46, 7894-7901.

(70) Hammouda, B.; Kim, M.-H. The empirical core-chain model. J. Mol. Liq. 2017, 247, 434-440.

(71) Small Angle X-ray Scattering; Glatter, O., Kratky, O., Eds.; Academic Press: London, 1982.

(72) Borisenko, K. B.; Rozsondai, B.; Hargittai, I. Molecular structure and intramolecular motion of hexamethyldisiloxane from gas-phase electron diffraction. J. Mol. Struct. 1997, 406, 137-144.

(73) Gilra, N.; Cohen, C.; Briber, R. M.; Bauer, B. J.; Hedden, R. C.; Panagiotopoulos, A. Z. A SANS Study of the Conformational Behavior of Linear Chains in Compressed and Uncompressed End-Linked Elastomers. Macromolecules 2001, 34, 7773-7782.

(74) onion-SasView 4.2.2 documentation. http:/ / www.sasview.org/ docs/user/models/onion.html (accessed $1^{\text {st }}$ November 2019).

(75) Washington, A. L.; Li, X.; Schofield, A. B.; Hong, K.; Fitzsimmons, M. R.; Dalgliesh, R.; Pynn, R. Inter-particle correlations in a hard-sphere colloidal suspension with polymer additives investigated by Spin Echo Small Angle Neutron Scattering (SESANS). Soft Matter 2014, 10, 3016-3026.

(76) Pekcan, O.; Winnik, M. A.; Croucher, M. D. A microphase model for sterically stabilized polymer colloids: Fluorescence energy transfer from naphthalene-labeled dispersions. J. Polym. Sci, Polym. Lett. Ed. 1983, 21, 1011-1018.

(77) Pekcan, O.; Winnik, M. A.; Egan, L.; Croucher, M. D. Luminescence techniques in polymer colloids. 1. Energy-transfer studies in nonaqueous dispersions. Macromolecules 1983, 16, 699-702.

(78) Pekcan, O.; Winnik, M. A.; Croucher, M. D. Phosphorescence from covalently labeled nonaqueous dispersions: Insights into the swelling of microdomains: 2 . Luminescence from polymer colloids. J. Colloid Interface Sci. 1983, 95, 420-427.

(79) Hammouda, B. Probing Nanoscale Structures-The SANS Toolbox. http://www.ncnr.nist.gov/staff/hammouda/the_SANS toolbox.pdf (accessed $1^{\text {st }}$ November 2019).

(80) Zhang, I.; Pinchaipat, R.; Wilding, N. B.; Faers, M. A.; Bartlett, P.; Evans, R.; Royall, C. P. Composition inversion in mixtures of binary colloids and polymer. J. Chem. Phys. 2018, 148, 184902.

(81) Smith, G. N.; Eastoe, J.; Grillo, I.; Hallett, J.; Peach, J.; Pegg, J. Surfactant-Induced Charging of Polydimethylsiloxane-Poly(methyl methacrylate) Latexes in Nonpolar Solvent; Institut Laue-Langevin (ILL), 2016 DOI: DOI: 10.5291/ILL-DATA.9-12-478). 